\title{
Seroepidemiology of Toxoplasma gondii Infection in Women of Reproductive Age: A Cross-Sectional Study in a Northwestern Mexican City
}

\author{
Cosme Alvarado-Esquivel ${ }^{\mathrm{a}, \mathrm{f}}$, Maria Alba Guadalupe Corella-Madueno ${ }^{\mathrm{b}}$, Jesus Hernandez-Tinoco ${ }^{\mathrm{c}}$, \\ Antonio Rascon-Careaga ${ }^{b}$, Luis Francisco Sanchez-Anguianoc ${ }^{c}$, Karla Guadalupe Martinez-Robinson ${ }^{\mathrm{d}}$, \\ Maria Lourdes Aldana-Madride, Trinidad Quizan-Plata ${ }^{\mathrm{b}}$, Maria Guadalupe Canez-Carrasco ${ }^{\mathrm{b}}$, \\ Cinthia Jhovanna Perez-Martinez ${ }^{\mathrm{b}}$
}

\begin{abstract}
Background: Through a cross-sectional survey, we determined the seroprevalence and correlates of Toxoplasma gondii (T. gondii) infection in women of reproductive age in Hermosillo City, Mexico.

Methods: We studied 445 women of reproductive age in Hermosillo City in the northwestern Mexican state of Sonora. Women were enrolled in the University of Sonora. Sera of women were examined for IgG and IgM antibodies to T. gondii by commercially available enzyme immunoassays. The association of $T$. gondii seropositivity with the characteristics of the pregnant women was determined by bivariate and multivariate analyses.
\end{abstract}

Results: Of the 445 women (mean age: $22.18 \pm 5.6$ years) studied, $16(3.6 \%)$ had IgG antibodies to T. gondii, and two (12.5\%) were also positive for IgM antibodies to $T$. gondii. Of the 16 anti-T. gondii IgGpositive women, six (37.5\%) had IgG levels higher than $150 \mathrm{IU} / \mathrm{mL}$, four $(25.0 \%)$ between 100 and $150 \mathrm{IU} / \mathrm{mL}$, and six (37.5\%) between 9 and $99 \mathrm{IU} / \mathrm{mL}$. Multivariate analysis of socio-demographic and behavioral variables showed that $T$. gondii seropositivity was associated with older age (odds ratio (OR): 5.30; 95\% confidence interval (CI): 1.37 - 20.50; $\mathrm{P}=0.01)$ and boar meat consumption (OR: 6.86; $95 \%$

Manuscript submitted November 27, 2017, accepted January 3, 2018

${ }^{a}$ Biomedical Research Laboratory, Faculty of Medicine and Nutrition, Juarez University of Durango State, Avenida Universidad S/N, 34000 Durango, Mexico

bDepartment of Chemical and Biological Sciences, University of Sonora, Boulevard Luis Encinas y Rosales S/N, 83000 Hermosillo, Mexico

'Institute for Scientific Research "Dr. Roberto Rivera-Damm", Juarez University of Durango State, Avenida Universidad S/N, 34000 Durango, Mexico ${ }^{\mathrm{d}}$ Center for Food and Development Research, Department of Technology of Food From Animal Origin, Carretera a la Victoria Km. 6, Hermosillo, Sonora, Mexico

eDepartment of Research and Postgraduate in Food, University of Sonora, Boulevard Luis Encinas y Rosales S/N, 83000 Hermosillo, Mexico

${ }^{f}$ Corresponding Author: Cosme Alvarado-Esquivel, Laboratorio de Investigacion Biomedica, Facultad de Medicina y Nutricion, Avenida Universidad S/N, 34000 Durango, Dgo, Mexico. Email: alvaradocosme@yahoo.com

doi: https://doi.org/10.14740/jocmr3284w
CI: 1.27 - 37.07; P = 0.02)

Conclusions: Women of reproductive age in Hermosillo City had a low seroprevalence of $T$. gondii infection. However, this finding indicates that most of these women were susceptible to a primary infection. Factors associated with T. gondii infection found in this study may be useful for the optimal planning of preventive measures against $T$. gondii infection and its sequelae.

Keywords: Toxoplasma; Women; Epidemiology; Seroprevalence; Risk factors; Cross-sectional study; Mexico

\section{Introduction}

The parasite Toxoplasma gondii (T. gondii) causes infections in humans and animals in all continents $[1,2]$. Infection with $T$. gondii is acquired by several routes, including uptake of oocysts released by felids [2,3], ingestion of raw or undercooked meat containing tissue cysts [4], congenitally [5], and organ transplant [6]. Infections with $T$. gondii are usually asymptomatic; however, some infected individuals may develop acute toxoplasmosis [7]. This disease may present as lymphadenopathy or chorioretinitis $[6,7]$, but a life-threatening meningoencephalitis may occur in immunocompromised patients [7]. On the other hand, primary infections with $T$. gondii in pregnant women are causes of miscarriages, stillbirths and congenital disease with eye and central nervous system involvement [7, 8]. Clinical manifestations of congenital disease can be present at birth or appear later in life $[9,10]$. Infection with $T$. gondii in most hosts leads to lifelong chronic infection and generates immunological memory responses that protect the host against new infections [11]. Infections with T. gondii in women immunized against this parasite rarely lead to congenital toxoplasmosis [12].

The seroepidemiology of $T$. gondii infection in women of reproductive age in Mexico has been scantly studied. In a national seroepidemiology survey about the prevalence of toxoplasmosis in Mexico in 1992, Velasco-Castrejon et al reported a high incidence of $T$. gondii infection in women of re- 
productive age [13]. We are not aware of any further study of T. gondii infection in women of reproductive age in Mexico. Therefore, we sought to determine the seroprevalence of $T$. gondii infection and factors associated with this infection in women of reproductive age in the northwestern Mexican city of Hermosillo.

\section{Materials and Methods}

\section{Study design and women studied}

Through a cross-sectional study design, we studied women of reproductive age in Hermosillo City in the northwestern Mexican state of Sonora. Women were enrolled in the University of Sonora from May 2015 to June 2017. As a strategy to enroll participants, university departments were visited, and women of reproductive age were informed about the project and invited to participate. Those women who agreed to participate were referred to the clinical laboratory of the university for blood sampling. Inclusion criteria were as follows: 1) female; 2) aged 13 - 46 years old; and 3) agreed to participate in the study. Occupation, socio-economic status, or educational level were not restrictive criteria for enrollment. In total, 445 women (mean age: $22.18 \pm 5.6$; range 13 - 46 years) were included in the study.

\section{Socio-demographic, clinical, and behavioral characteris- tics of the women}

Socio-demographic, clinical, and behavioral characteristics of the women were obtained with the aid of a standardized questionnaire. Socio-demographic items were age, birthplace, residence, occupation, educational level, and socio-economic status. Clinical data included obstetric history (number of pregnancies, deliveries, cesarean sections, and miscarriages), and history of blood transfusions or transplants. Behavioral items included presence of cats at home, cats in the neighborhood, cleaning cat feces, raising farm animals, foreign travel, consumption of raw meat, type of meat consumed (pork, lamb, beef, goat, boar, chicken, turkey, rabbit, deer, squirrel, horse, or other), eating away from home (in restaurants and fast food outlets), consumption of cured meat (chorizo, ham, sausages or salami), consumption of unwashed raw vegetables or fruits, consumption of untreated water or unpasteurized milk, and soil contact (gardening or agriculture).

\section{Detection of anti- $T$. gondii antibodies}

A serum sample was obtained from each woman and stored at $-20{ }^{\circ} \mathrm{C}$ until analyzed. All serum samples were analyzed for IgG antibodies to $T$. gondii by a commercially available enzyme immunoassay "Toxoplasma IgG" kit (Diagnostic Automation/Cortez Diagnostics Inc., Woodland Hills, CA, USA). Serum samples positive for IgG antibodies to $T$. gondii were further tested for IgM antibodies to T. gondii by a commercially available enzyme immunoassay "Toxoplasma IgM" kit (Di- agnostic Automation/Cortez Diagnostics Inc.). All tests were performed following manufacturer instructions. Positive and negative controls for IgG and IgM were included in each run.

\section{Statistical analysis}

We performed the statistical analysis with the aid of Epi Info version 7 and SPSS version 15.0 software. For calculation of the sample size, we used: 1) a reference seroprevalence of $13.5 \%$ [14], as the expected frequency for the factor under study, 2) 250,000 , as the population size from which the sample was selected, 3) confidence limits of 3.5\%, and 4) a $95 \%$ confidence level. The result of the sample size calculation was 366 subjects. We used Pearson's Chi-square test or the Fisher's exact test (when values were less than 5) for comparison of the frequencies among groups. Multivariate analysis was used to determine the association between $T$. gondii seropositivity and the socio-demographic, and behavioral characteristics of the women. Only variables with a $\mathrm{P}$ value $\leq 0.10$ obtained in the bivariate analysis were further analyzed by multivariate analysis. To avoid bias, clinical data were not included in the multivariate analysis. We calculated the odds ratios (ORs) and 95\% confidence intervals (CIs) by logistic regression analysis using the Enter method. A P value $<0.05$ was considered significant.

\section{Ethics aspects}

This study was approved by The Institutional Ethics Committee of the University of Sonora, Mexico. The purpose and procedures of the study were explained to all participants. Additionally, written informed consent was obtained from all participants.

\section{Results}

Of the 445 women studied, $16(3.6 \%)$ had $\mathrm{IgG}$ antibodies to $T$. gondii, and two (12.5\%) of these participants were also positive for IgM antibodies to $T$. gondii by enzyme immunoassays. Of the 16 anti-T. gondii IgG positive women, six (37.5\%) had IgG levels higher than $150 \mathrm{IU} / \mathrm{mL}$, four $(25.0 \%)$ between 100 and $150 \mathrm{IU} / \mathrm{mL}$, and six (37.5\%) between 9 and $99 \mathrm{IU} / \mathrm{mL}$. The socio-demographic characteristics of the women and correlation with $T$. gondii IgG seropositivity are shown in Table 1. The variables "age groups", "occupation" and "socioeconomic level" showed $\mathrm{P}$ values $\leq 0.10$ by bivariate analysis. Other socio-demographic variables of women, including birthplace, residence, and educational level showed $\mathrm{P}$ values higher than 0.10 by bivariate analysis.

Concerning behavioral characteristics, bivariate analysis showed that the variables "sheep meat consumption" and "boar meat consumption" showed $\mathrm{P}$ values $\leq 0.10$. Other behavioral variables, including contact with cats, raising farm animals, foreign travel, consumption of raw meat, consumption of pork, beef, venison or meat of goat, chicken, turkey, rabbit, squirrel, horse, or other animals, eating away from home, consumption 
Table 1. Socio-Demographic Characteristics of Women and Prevalence of T. gondii Infection

\begin{tabular}{|c|c|c|c|c|}
\hline \multirow{2}{*}{ Characteristics } & \multirow{2}{*}{ No. } & \multicolumn{2}{|c|}{ Prevalence of $T$. gondii infection } & \multirow{2}{*}{ P value } \\
\hline & & No. & $\%$ & \\
\hline \multicolumn{5}{|l|}{ Age group (years) } \\
\hline $13-30$ & 399 & 10 & 2.5 & 0.003 \\
\hline $31-46$ & 46 & 6 & 13.0 & \\
\hline \multicolumn{5}{|l|}{ Birth place } \\
\hline Sonora State & 440 & 16 & 3.6 & 0.91 \\
\hline Other Mexican State & 4 & 0 & 0.0 & \\
\hline Abroad & 1 & 0 & 0.0 & \\
\hline \multicolumn{5}{|l|}{ Residence place } \\
\hline Sonora State & 441 & 16 & 3.6 & 1.00 \\
\hline Other Mexican State & 4 & 0 & 0.0 & \\
\hline \multicolumn{5}{|l|}{ Residence area } \\
\hline Urban & 392 & 12 & 3.1 & 0.18 \\
\hline Suburban & 8 & 1 & 12.5 & \\
\hline Rural & 45 & 3 & 6.7 & \\
\hline \multicolumn{5}{|l|}{ Educational level } \\
\hline No education & 17 & 0 & 0.0 & 0.74 \\
\hline $1-6$ years & 106 & 5 & 4.7 & \\
\hline $7-12$ years & 317 & 11 & 3.5 & \\
\hline$>12$ years & 5 & 0 & 0.0 & \\
\hline \multicolumn{5}{|l|}{ Occupation } \\
\hline Laborer $^{\mathrm{a}}$ & 32 & 4 & 12.5 & 0.02 \\
\hline Non-laborer ${ }^{\mathrm{b}}$ & 413 & 12 & 2.9 & \\
\hline \multicolumn{5}{|l|}{ Socio-economic level } \\
\hline Low & 6 & 1 & 16.7 & 0.07 \\
\hline Medium & 352 & 10 & 2.8 & \\
\hline High & 87 & 5 & 5.7 & \\
\hline
\end{tabular}

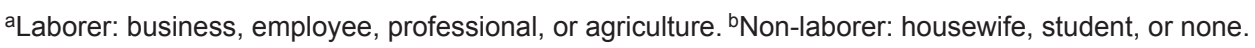

of cured meat, consumption of unwashed raw vegetables or fruits, consumption of untreated water or unpasteurized milk, and soil contact showed $\mathrm{P}$ values higher than 0.10 by bivariate analysis. Table 2 shows a selection of behavioral characteristics of the women and the correlation with $T$. gondii IgG seropositivity. Multivariate analysis of socio-demographic, and behavioral variables with $\mathrm{P}$ values $\leq 0.10$ obtained in the bivariate analysis showed that $T$. gondii seropositivity was associated with older age (OR: 5.30; 95\% CI: 1.37 - 20.50; P=0.01), and boar meat consumption (OR: 6.86; 95\% CI: 1.27 - 37.07; $\mathrm{P}=0.02$ ). Table 3 shows the results of the regression analysis.

With respect to clinical data, bivariate analysis showed that seropositivity to $T$. gondii was associated with the variables "history of pregnancy" $(\mathrm{P}=0.02)$ and "history of miscarriage" $(\mathrm{P}=0.03)$. Whereas other clinical variables, including history of deliveries, cesarean sections, or blood transfusion, did not show an association with $T$. gondii seropositivity. None of the women studied had a history of organ transplant. Results of the correlation between clinical characteristics and seropositivity to $T$. gondii are shown in Table 4.

\section{Discussion}

The seroepidemiology of $T$. gondii infection in women of reproductive age in Mexico is largely unknown. Epidemiological studies on this infection in women in Mexico have focused on pregnant women. Therefore, in this study, we aimed to determine the seroprevalence and determinants of $T$. gondii infection in women of reproductive age in the northwestern Mexican city of Hermosillo. In this study, we found a $3.6 \%$ seroprevalence of $T$. gondii infection in the women studied. We are aware of only one previous report on the seroepidemiology of $T$. gondii infection in women of reproductive age in Mexico. In a national serosurvey of $T$. gondii infection in all 32 states of this country, Velasco-Castrejon et al found a 
Table 2. Bivariate Analysis of Selected Putative Risk Factors for Infection With T. gondii in Women

\begin{tabular}{|c|c|c|c|c|}
\hline \multirow{2}{*}{ Characteristics } & \multirow{2}{*}{ No. of women tested } & \multicolumn{2}{|c|}{ Prevalence of $T$. gondii infection } & \multirow{2}{*}{ P value } \\
\hline & & No. & $\%$ & \\
\hline \multicolumn{5}{|l|}{ Cats at home } \\
\hline Yes & 154 & 6 & 3.9 & 0.80 \\
\hline \multicolumn{5}{|c|}{ Cleaning cat excrement } \\
\hline Yes & 58 & 1 & 1.7 & 0.70 \\
\hline No & 387 & 15 & 3.9 & \\
\hline Yes & 291 & 12 & 4.1 & 0.41 \\
\hline No & 154 & 4 & 2.6 & \\
\hline \multicolumn{5}{|l|}{ National trips } \\
\hline Yes & 274 & 7 & 2.6 & 0.21 \\
\hline No & 169 & 8 & 4.7 & \\
\hline Yes & 418 & 16 & 3.8 & 0.61 \\
\hline No & 27 & 0 & 0.0 & \\
\hline \multicolumn{5}{|c|}{ Goat meat consumption } \\
\hline Yes & 25 & 2 & 8.0 & 0.22 \\
\hline No & 420 & 14 & 3.3 & \\
\hline \multicolumn{5}{|c|}{ Sheep meat consumption } \\
\hline Yes & 67 & 5 & 7.5 & 0.07 \\
\hline No & 378 & 11 & 2.9 & \\
\hline \multicolumn{5}{|c|}{ Boar meat consumption } \\
\hline Yes & 14 & 2 & 14.3 & 0.08 \\
\hline No & 431 & 14 & 3.2 & \\
\hline Yes & 6 & 0 & 0.0 & 1.00 \\
\hline No & 439 & 16 & 3.6 & \\
\hline \multicolumn{5}{|c|}{ Cow raw milk consumption } \\
\hline Yes & 121 & 3 & 2.5 & 0.57 \\
\hline No & 324 & 13 & 4.0 & \\
\hline \multicolumn{5}{|c|}{ Goat raw milk consumption } \\
\hline Yes & 21 & 1 & 4.8 & 0.54 \\
\hline No & 424 & 15 & 3.5 & \\
\hline \multicolumn{5}{|c|}{$\begin{array}{l}\text { Unwashed raw vegetables } \\
\text { consumption }\end{array}$} \\
\hline Yes & 66 & 0 & 0.0 & 0.14 \\
\hline No & 379 & 16 & 4.2 & \\
\hline \multicolumn{5}{|c|}{ Untreated water consumption } \\
\hline Yes & 37 & 0 & 0.0 & 0.38 \\
\hline No & 408 & 16 & 3.9 & \\
\hline Soil contact & & & & \\
\hline Yes & 149 & 7 & 4.7 & 0.37 \\
\hline No & 296 & 9 & 3.0 & \\
\hline
\end{tabular}


Table 3. Multivariate Analysis of Selected Characteristics of Women and Their Association With T. gondii Infection

\begin{tabular}{llll}
\hline Characteristics & Odds ratio & $\mathbf{9 5 \%}$ confidence interval & P value \\
\hline Older age (31 - 46 years old) & 5.30 & $1.37-20.50$ & 0.01 \\
Occupation (laborer) & 1.85 & $0.40-8.52$ & 0.42 \\
Socio-economic level (low) & 1.45 & $0.46-4.48$ & 0.51 \\
Sheep meat consumption (yes) & 2.35 & $0.74-7.42$ & 0.14 \\
Boar meat consumption (yes) & 6.86 & $1.27-37.07$ & 0.02 \\
\hline
\end{tabular}

$33.4 \%$ mean seroprevalence of $T$. gondii infection in women aged 15 - 39 years old using indirect immunofluorescence assay with a cut-off titer of $1: 128$ [13]. Therefore, the $3.6 \%$ seroprevalence found in women of reproductive age in Hermosillo City is much lower than the $33.4 \%$ mean seroprevalence of T. gondii infection in women aged 15 - 39 years found in the national survey [13]. However, comparison of these seroprevalences should be cautious, since there were differences in age and serological test for detection of $T$. gondii infection among the studies. We studied women aged 13 - 46 years old, whereas researchers of the national survey studied women aged 15 39 years old. With respect to the tests for detection of anti- $T$. gondii antibodies, we used a commercially available enzyme immune assay, while researchers of the national survey used an indirect immunofluorescence assay [13]. The results of the national survey were published approximately 25 years ago, and the low seroprevalence of $T$. gondii infection found in women in Hermosillo City may reflect an improvement of hygiene and sanitation measures against $T$. gondii exposure in recent times. In an international context, the seroprevalence of $T$. gondii infection in women in Hermosillo City, Sonora, is lower than the seroprevalence reported in women of reproductive age in central Italy (23.6\%) [15], Senegal (40.2\%) [16],
Poland (59\%) [17], Turkey (58.3\%) [18], Cote de Ivoire (60\%) [19], Yugoslavia (77\%) [20], and Israel (15.1-72.3\%) [21]. We did not find in the literature any reports of seroprevalence of T. gondii infection in women of reproductive age equal to or lower than that obtained in our study. It is unclear why women in Hermosillo City, Mexico, had a lower seroprevalence of $T$. gondii infection than that reported in women of reproductive age in other countries. Differences in environmental characteristics among the studies might explain the differences in seroprevalence. Hermosillo City has a hot desert climate, and hot and dry climate conditions are not favorable for $T$. gondii [21]. In a study about quantification of oocyst viability in soil over time, oocysts survived longer in damp conditions than in dry conditions [22]. Additionally, lower seroprevalence of $T$. gondii infection in dry rather than humid climates has been observed in human populations [21, 23] and in rabbits [24].

We searched for socio-demographic and behavioral determinants associated with $T$. gondii infection. Bivariate analysis showed that laborers had a higher seroprevalence of $T$. gondii infection than non-laborers. It is possible that laborers had more exposure factors for T. gondii infection, i.e. soil contact, older age, etc., than non-laborers. Regression analysis showed that $T$. gondii seropositivity was associated with older age and

Table 4. Bivariate Analysis of Clinical Data of Women and Seroprevalence of Infection With T. gondii

\begin{tabular}{|c|c|c|c|c|}
\hline \multirow{2}{*}{ Characteristics } & \multirow{2}{*}{ No. of women tested } & \multicolumn{2}{|c|}{ Prevalence of $T$. gondii infection } & \multirow{2}{*}{ P value } \\
\hline & & No. & $\%$ & \\
\hline \multicolumn{5}{|l|}{ Pregnancies } \\
\hline No & 379 & 10 & 2.6 & \\
\hline \multicolumn{5}{|l|}{ Deliveries } \\
\hline No & 393 & 12 & 3.1 & \\
\hline \multicolumn{5}{|l|}{ Cesarean sections } \\
\hline Yes & 30 & 3 & 10.0 & 0.08 \\
\hline No & 415 & 13 & 3.1 & \\
\hline \multicolumn{5}{|l|}{ Miscarriages } \\
\hline Yes & 14 & 0 & 0 & 1.00 \\
\hline No & 431 & 16 & 3.7 & \\
\hline
\end{tabular}


boar meat consumption. Concerning the link between infection and age, our results agree with other studies. Infection with $T$. gondii has been associated with increasing age in women of reproductive age in central Italy [15], Turkey [18], and Yugoslavia [20]. With respect to the link between infection and boar meat consumption, our results are in line with those reported in previous studies in northern Mexico. We previously found an association between $T$. gondii infection and boar meat consumption in several populations in Durango State, Mexico, including miners [25], elderly people [26], patients with work accidents [27], and the general urban population [28]. The results thus indicate that boar meat consumption is an important factor for T. gondii infection in northern Mexico.

Concerning clinical characteristics of women, in the present study, we found that the frequency of $T$. gondii exposure was significantly higher in women with a history of pregnancy and miscarriage. The association between infection and pregnancy might be related with age, since older women are more likely to have had multiple pregnancies in their lives than younger women. However, unknown factors might have influenced the seroprevalence of $T$. gondii infection in women with a history of pregnancy, and further research to elucidate the cause for $T$. gondii exposure in these women should be conducted. The finding of an association between infection and miscarriage agrees with those reported in previous studies in northern Mexico. This association was found in women of the ethnic group of Yoremes in the same state of Sonora [29]. Additionally, an association between high anti-T. gondii IgG antibody levels and miscarriages was found in subjects applying for medical certificates in Durango City, Mexico [30]. These serological findings suggest that $T$. gondii infection may be causing miscarriages in northern Mexico.

The present study has some limitations, including a low seroprevalence of $T$. gondii infection in the women studied and sampling of women in only one institution. Further studies with a larger sample size, performed in several institutions, to confirm our results are needed.

\section{Conclusions}

Women of reproductive age in Hermosillo City had a low seroprevalence of $T$. gondii infection. However, this finding indicates that most of these women were susceptible to a primary infection. Factors associated with $T$. gondii infection found in this study may be useful for optimal planning of preventive measures against $T$. gondii infection and its sequelae.

\section{Acknowledgments}

This study was supported by Juarez University of Durango State, and University of Sonora, Hermosillo, Sonora, Mexico.

\section{Competing Interests}

The authors declare that no competing interests exist.

\section{References}

1. Hill DE, Dubey JP. Toxoplasma gondii as a parasite in food: analysis and control. Microbiol Spectr. 2016;4(4):PFS-0011-2015.

2. Schluter D, Daubener W, Schares G, Gross U, Pleyer U, Luder C. Animals are key to human toxoplasmosis. Int J Med Microbiol. 2014;304(7):917-929.

3. Dabritz HA, Conrad PA. Cats and Toxoplasma: implications for public health. Zoonoses Public Health. 2010;57(1):34-52.

4. Pereira KS, Franco RM, Leal DA. Transmission of toxoplasmosis (Toxoplasma gondii) by foods. Adv Food Nutr Res. 2010;60:1-19.

5. Elmore SA, Jones JL, Conrad PA, Patton S, Lindsay DS, Dubey JP. Toxoplasma gondii: epidemiology, feline clinical aspects, and prevention. Trends Parasitol. 2010;26(4):190-196.

6. Khurana S, Batra N. Toxoplasmosis in organ transplant recipients: Evaluation, implication, and prevention. Trop Parasitol. 2016;6(2):123-128.

7. Montoya JG, Liesenfeld O. Toxoplasmosis. Lancet. 2004;363(9425):1965-1976.

8. Singh S. Congenital toxoplasmosis: Clinical features, outcomes, treatment, and prevention. Trop Parasitol. 2016;6(2):113-122.

9. Vutova K, Peicheva Z, Popova A, Markova V, Mincheva N, Todorov T. Congenital toxoplasmosis: eye manifestations in infants and children. Ann Trop Paediatr. 2002;22(3):213-218

10. Moncada PA, Montoya JG. Toxoplasmosis in the fetus and newborn: an update on prevalence, diagnosis and treatment. Expert Rev Anti Infect Ther. 2012;10(7):815828.

11. Jensen KD, Camejo A, Melo MB, Cordeiro C, Julien L, Grotenbreg GM, Frickel EM, et al. Toxoplasma gondii superinfection and virulence during secondary infection correlate with the exact ROP5/ROP18 allelic combination. MBio. 2015;6(2):e02280.

12. Lebas F, Ducrocq S, Mucignat V, Paris L, Megier P, Baudon JJ, Gold F. [Congenital toxoplasmosis: a new case of infection during pregnancy in an previously immunized and immunocompetent woman]. Arch Pediatr. 2004;11(8):926-928.

13. Velasco-Castrejon O, Salvatierra-Izaba B, Valdespino JL, Sedano-Lara AM, Galindo-Virgen S, Magos C, Llausas A, et al. [Seroepidemiology of toxoplasmosis in Mexico]. Salud Publica Mex. 1992;34(2):222-229.

14. Alvarado-Esquivel C, Rascon-Careaga A, HernandezTinoco J, Corella-Madueno MA, Sanchez-Anguiano LF, Aldana-Madrid ML, Velasquez-Vega E, et al. Seroprevalence and associated risk factors for Toxoplasma gondii infection in healthy blood donors: a cross-sectional study in Sonora, Mexico. Biomed Res Int. 2016;2016:9597276.

15. Mosti M, Pinto B, Giromella A, Fabiani S, Cristofani R, Panichi M, Bruschi F. A 4-year evaluation of toxoplasmosis seroprevalence in the general population and in women of reproductive age in central Italy. Epidemiol Infect. 
2013;141(10):2192-2195.

16. Faye O, Leye A, Dieng Y, Richard-Lenoble D, Diallo S. [Toxoplasmosis in Dakar. Seroepidemiologic sampling of 353 women of reproductive age]. Bull Soc Pathol Exot. 1998;91(3):249-250.

17. Kurnatowska A, Tomczewska I. [Prevalence of Toxoplasma gondii and analysis of specific immunoglobulins concentration in serum of women during the reproductive period in a sample of Wloclawek population]. Wiad Parazytol. 2001;46(Suppl 1):77-82.

18. Yentur Doni N, Simsek Z, Gurses G, Yildiz Zeyrek F, Demir C. Prevalence and associated risk factors of Toxoplasma gondii in female farmworkers of southeastern Turkey. J Infect Dev Ctries. 2015;9(1):87-93.

19. Adou-Bryn KD, Ouhon J, Nemer J, Yapo CG, Assoumou A. [Serological survey of acquired toxoplasmosis in women of child-bearing age in Yopougon (Abidjan, Cote d'Ivoire)]. Bull Soc Pathol Exot. 2004;97(5):345-348.

20. Bobic B, Jevremovic I, Marinkovic J, Sibalic D, Djurkovic-Djakovic O. Risk factors for Toxoplasma infection in a reproductive age female population in the area of Belgrade, Yugoslavia. Eur J Epidemiol. 1998;14(6):605-610.

21. Markovich MP, Shohat T, Riklis I, Avni R, YujelevskiRozenblit D, Bassal R, Cohen D, et al. Seroepidemiology of Toxoplasma gondii infection in the Israeli population. Epidemiol Infect. 2014;142(1):149-155.

22. Lelu M, Villena I, Darde ML, Aubert D, Geers R, Dupuis E, Marnef F, et al. Quantitative estimation of the viability of Toxoplasma gondii oocysts in soil. Appl Environ Microbiol. 2012;78(15):5127-5132.

23. Assmar M, Amirkhani A, Piazak N, Hovanesian A, Kooloobandi A, Etessami R. [Toxoplasmosis in Iran. Results of a seroepidemiological study]. Bull Soc Pathol Exot. 1997;90(1):19-21.

24. Almeria S, Calvete C, Pages A, Gauss C, Dubey JP. Factors affecting the seroprevalence of Toxoplasma gondii infection in wild rabbits (Oryctolagus cuniculus) from Spain. Vet Parasitol. 2004;123(3-4):265-270.

25. Alvarado-Esquivel C, Pacheco-Vega SJ, HernandezTinoco J, Berumen-Segovia LO, Sanchez-Anguiano LF, Estrada-Martinez S, Sandoval-Carrillo AA, et al. High Prevalence of Toxoplasma gondii Infection in Miners: A Case-Control Study in Rural Durango, Mexico. J Clin Med Res. 2016;8(12):870-877.

26. Alvarado-Esquivel C, Liesenfeld O, Burciaga-Lopez BD, Ramos-Nevarez A, Estrada-Martinez S, CerrilloSoto SM, Carrete-Ramirez FA, et al. Seroepidemiology of Toxoplasma gondii infection in elderly people in a northern Mexican city. Vector Borne Zoonotic Dis. 2012;12(7):568-574.

27. Alvarado-Esquivel C, Torres-Castorena A, Liesenfeld O, Estrada-Martinez S, Urbina-Alvarez JD. High seroprevalence of Toxoplasma gondii infection in a subset of Mexican patients with work accidents and low socioeconomic status. Parasit Vectors. 2012;5:13.

28. Alvarado-Esquivel C, Estrada-Martinez S, Pizarro-Villalobos H, Arce-Quinones M, Liesenfeld O, Dubey JP. Seroepidemiology of Toxoplasma gondii infection in general population in a northern Mexican city. J Parasitol. 2011;97(1):40-43.

29. Alvarado-Esquivel C, Rascon-Careaga A, HernandezTinoco J, Corella-Madueno MA, Sanchez-Anguiano LF, Aldana-Madrid ML, Almada-Balderrama GJ, et al. Seroprevalence and correlates of Toxoplasma gondii infection in Yoremes (Mayos) in Mexico: a cross-sectional study. BMJ Open. 2016;6(5):e010218.

30. Alvarado-Esquivel C, Sanchez-Anguiano LF, Hernandez-Tinoco J, Pulido-Montoya RA, Acosta-Rojas G, Estrada-Martinez S, Perez-Alamos AR, et al. Seroepidemiology of Toxoplasma gondii infection in people applying for medical certificates. Eur J Microbiol Immunol (Bp). 2016;6(2):90-98. 